\title{
Neuropsychological Deficits Are Correlated with Frontal Hypometabolism in Positron Emission Tomography Studies of Older Alcoholic Patients
}

\author{
Kenneth M. Adams, Sid Gilman, Robert A. Koeppe, Karen J. Kluin, James A. Brunberg, \\ Duane Dede, Stanley Berent, and Phillip D. Kroll
}

\begin{abstract}
In an extension of previous work, we studied the behavioral correlates of medial trontal lobe glucose hypometabolism in chronically alcohol-dependent patients. Thirty-one male patients who were detoxified, medically stable, and free of other central nervous system risk factors for neuropsychological impairment were examined with (1) anatomic imaging (CT or MR), (2) functional imaging with $\left[{ }^{18} \mathrm{~F}\right]$ fluorodeoxyglucose $\left({ }^{18} \mathrm{~F}-\mathrm{FDG}\right)$ and positron emission tomography (PET), and (3) a battery of neuropsychological tests, including two measures of abstraction known to be generally sensitive to frontal lobe disease or dysfunction [the Wisconsin Card Sorting Test (WCST) and the Halstead Category Test (HCT)]. ${ }^{18}$ F-FDG PET data from 18 age- and sex-matched normal control subjects were used for comparison. All patients met criteria for severe alcohol dependence and for at least a mild degree of alcoholic-induced cognitive impairment. Although the mean IQ level of the alcoholic patients was in the average range, the concepts attained and the error scores on the WCST and HCT were significantly impaired in comparison with established norms. Local cerebral metabolic rate for glucose (LCMRglc) was significantly decreased in a sagittal strip of the medial frontal cortex in the alcoholic patients as compared with the normal controls. Comparison of data from PET scans and anatomic images indicated that the reduced LCMRglc could not be attributed to reduced amounts of tissue alone. A statistically significant relationship was found between LCMRglc in the medial frontal region of the cerebral cortex and performance on the WCST, but not the HCT. These findings suggest that chronic alcohol intake results in impaired function of cerebral tissue in the medial frontal region. The impairment pertains both to tissue metabolic rates and the behavioral correlates of these rates.
\end{abstract}

Key Words: Alcoholism, Neuropsychology, Positron Emission Tomography, Imaging.

CHRONIC CONSUMPTION OF alcoholic beverages in association with malnutrition may result in persisting neuropsychological deficits that often include intellectual, memory, higher reasoning, and attentional skills. ${ }^{1-3}$ Among the deficits of higher reasoning are im-

From the Psychology, Neurology, and Psychiatry Services, Veteran's Affairs Medical Center; and the Departments of Psychiatry, Neurology, Internal Medicine, Physical Medicine and Rehabilitation, and Radiology, The University of Michigan Medical Center, Ann Arbor, MI 48109-0704.

Received for publication March 9, 1992; accepted August 28, 1992

This work was supported in part by grants from the National Institute on Alcohol Abuse and Alcoholism (AA07378), the National Institutes of Health (NS15655), and a sharing agreement between the Ann Arbor Veteran's Affairs Medical Center and The University of Michigan.

Reprint requests: Kenneth M. Adams, Ph.D. Department of Psychiatry (Box 0704), University of Michigan Medical Center, Ann Arbor, MI 48109-0704.

Copyright $@ 1993$ by The Research Society on Alcoholism. Alcohol Clin Exp Res. Vol 17. No 2, 1993: pp 205-210 pairments in conceptual skills and difficulty with "abstract" thinking. ${ }^{4}$ The regions of the central nervous system responsible for these deficits in living subjects have not been precisely determined. Studies correlating structural brain degeneration on computed tomography (CT) brain scan with neuropsychological impairment have produced inconsistent results. ${ }^{5}$ Better visualization of abnormal tissue structures may be possible with magnetic resonance (MR) imaging. ${ }^{6}$ However, reliable clinical correlation between structural imaging techniques and validated neuropsychological tests has not yet been achieved in the individual case.

In two previous studies, ${ }^{7,8}$ examination of chronically alcohol-dependent patients with $\left[{ }^{18} \mathrm{~F}\right]$ fluorodeoxyglucose $\left({ }^{18} \mathrm{~F}-\mathrm{FDG}\right)$ and positron emission tomography (PET) revealed bilateral hypometabolism in the rostral medial frontal region of the cerebral cortex when compared with age-matched control subjects. This finding suggested that chronic intake of alcohol, perhaps in association with episodic malnutrition, specifically impairs the function of this region of the central nervous system. We now report the results of our studies of chronically alcohol-dependent patients using ${ }^{18} \mathrm{~F}$-FDG PET and neuropsychological tests designed to examine the function of this part of the cerebral cortex. We used anatomic imaging with MR or CT scans for correlations of anatomic changes with behavioral and PET data. The anatomic imaging was also used to assess and correct statistically for the degree of local cerebral atrophy in the analysis of the PET data.

We also explored some of the clinical features of alcoholism (age, years of heavy drinking, and peak drinking consumption) that might be related to the medial frontal hypometabolism. We correlated these factors with the results of selected neuropsychological tests, structural imaging data (CT or MR), and functional (PET) imaging data.

\section{METHOD}

\section{Subjects}

Thirty-one male veteran patients were recruited for this study from the Alcohol Treatment Unit of the Ann Arbor Veteran's Affairs Medical Center. Twenty-seven were right-handed and four were left-handed by behavioral test and preference (see Table 1).

All patients studied would be characterized as severe and chronic 
Table 1. Characteristics of the 31 Male Chronically Alcohol-Dependent Patients in This Study

\begin{tabular}{lcc}
\hline & Mean & so \\
\hline Age in years (range 42-78 years) & 51.1 & 8.9 \\
Education in years & 12.8 & 2.5 \\
Years of heavy drinking & 21.7 & 7.9 \\
Average no. of drinks* daily during & 14.3 & 5.4 \\
$\quad$ heavy drinking & 24.3 & 9.3 \\
$\begin{array}{l}\text { Maximum no. of drinks daily during } \\
\text { heavy drinking }\end{array}$ & & \\
\hline
\end{tabular}

$n=31$.

" A drink is defined as $4 \mathrm{oz}$ of 80 proof alcohol or its equivalent.

cases of alcoholism by any widely used nosology (e.g., Diagnostic and Statistical Manual (DSM) or International Classification of Diseases (ICD), etc). Each of the patients exceeded a weekly intake of at least 560 $\mathrm{g}$ of ethanol over two of the preceding 3 years. In all cases, the patients substantially exceeded this intake for a number of years prior to their entry into the study. The patients averaged at least one previous hospitalization for alcoholism, and 25 of 31 patients had achieved sobriety for only 1 month or more from the time they began drinking constantly, but had relapsed. These data characterize the patients as being "heavy" drinkers in both duration and intensity of alcohol intake. ${ }^{9}$

All patients received psychiatric examinations and all met formal diagnostic criteria for Alcohol Dependence; and all fit criteria for at least a mild degree of Dementia Associated with Alcoholism as defined in DSM-III-R. ${ }^{10}$ Nine patients had evidence of alcoholic cerebellar degeneration as determined by history, neurological examination, anatomical imaging study, and positron emission tomography. ${ }^{8}$ All patients were examined by a neurologist (S.G.), who obtained a history and conducted a physical and neurological examination.

The patients were selected to exclude those with any history of primary polydrug abuse, as reflected in the following quantitative criteria to define abuse as set by the National Institute on Drug Abuse in its National Collaborative Study of Polydrug Abuse ${ }^{11}$ : (1) no recreational injection of any drug at any time in their lives; (2) no more than six uses of marijuana in the year preceding entry to the study; (3) no more than 20 total occasions of hallucinogen use (including PCP); (4) no more than 5 noninjectable uses of heroin/opium in the preceding year; $(5)$ no more than 100 pills, tablets, or capsules of synthetic narcotics in the preceding year; (6) no more than 15 nonpharmaceutical uses of amphetamines or cocaine ever in their lives; (7) no more than 90 uses of pharmaceutical amphetamines or methylphenidate in their lives; (8) no more than 30 (nonprescription) uses of minor tranquilizers (e.g., diazepam and chlordiazepoxide) in the year preceding testing; (9) no more than 60 uses of sedative-hypnotics in the year prior to the study; and (10) no other use of exotic chemical comforts (e.g., amyl nitrate) in context. All patients met the criteria for non-polydrug status.

Patients with neurological disorders of any kind, apart from alcoholrelated, were excluded at recruitment. Other exclusion criteria included closed head injury with loss of consciousness, stroke, and other risk factors, such as birth complications, learning disorder, or other acquired or developmental disease, disorder or trauma. ${ }^{12}$

The patients were studied while in the hospital within in an alcohol treatment unit after a mean abstinence of 34 days and no less than 14 days. Most had a clinical history of malnutrition at some time in their drinking careers, but had been provided with standard nutritional intake and multiple vitamin therapy on admission to hospital. A dietitian reviewed each patient's nutrition, and all patients studied were consuming a normal diet for at least 3 weeks prior to their scans. Blood and urine examinations performed within seven days of the PET scan showed no evidence of ketosis or abnormal glucose levels. During the period of study, the patients were taking no medications other than disulfram known to affect central nervous system function.

Eighteen normal control male subjects were selected for comparison of ${ }^{18}$ F-FDG PET data. These subjects were nonpatient volunteers recruited and screened for inclusion as controls in various studies ongoing at the University of Michigan PET Center. They were comparable in terms of age (average age: 55 years, SD 9.1 years; range 43 to 81 years). None had self-reported drinking problems, previous diagnosis of alcoholism, or treatment for alcohol dependency.

\section{Neuropsychological Testing}

Neuropsychological examinations were performed on each patient. The examinations took place in a quiet, dedicated laboratory near the patient's hospital unit. Testing was accomplished only when subjects were completely willing to cooperate.

Tests were administered by experienced examiners who were naive as to the specific hypotheses of the research project. Each subject completed a protocol consisting of the Wechsler Adult Intelligence Scale-Revised and a modified and extended Halstead-Reitan Neuropsychological Test Battery. ${ }^{8}$ Of particular focus in this investigation were two test of abstraction and problem-solving, the Wisconsin Card Sorting Test (WCST) ${ }^{13}$ and the Halstead Category Test (HCT),${ }^{14}$ because these measures have been most closely associated with frontal lobe disorders. ${ }^{15}$

\section{PET Scans}

PET scans were performed with two scanners. The initial 14 subjects were studied with a TCC PCT $4600 \mathrm{~A}$ tomograph, ${ }^{15}$ and the remaining scans were accomplished with a Siemens 931-08/12 whole-body, multislice PET tomograph. The technical details of the TCC tomograph have been described previously. ${ }^{15}$ The Siemens tomograph operates with bismuth germanate detectors arranged in an eight crystal by four crystal matrix over four photomultiplier tubes. The individual crystal dimensions are $5.6 \mathrm{~mm}$ wide $\times 12.9 \mathrm{~mm}$ high $\times 30 \mathrm{~mm}$ deep. Four crystal/ tube arrangements of blocks are grouped into a bucket, which is a subunit of the tomograph. Sixteen buckets make up four rings for a total of 2048 detectors. The scanner has eight rings or 4096 total detectors. Coincidence resolving time is 5 nsec [full width at half-maximum (FWHM)]. Tomographic slice data were obtained from each ring and from between rings to yield 15 simultaneous image slices. Scanner resolution is $5.5 \mathrm{~mm}$ FWHM within the tomographic plane at $9 \mathrm{~cm}$ from the axis. This figure is degraded by approximately $15 \%$ at $20 \mathrm{~cm}$ from the axis. Z-axis resolution is 5.5 to $6.0 \mathrm{~mm}$ FWHM for each slide. Ray/angle information is bussed from each bucket to a VME 68000-based real time sorter (RTS). The data are buffered within the RTS to permit simultaneous acquisition and Direct Memory Access (DMA) transfer to the reconstruction hardware. A Digital Equipment Corporation uVAX provides system management of the analugic AP-400 Backprojector for image reconstruction, the DeAnza ID-5000 for image display, and an International Imaging System IVAS for PET data analysis, as well as for disk storage and DMA input/output to the RTS. The tomograph has a dedicated UVAX for data acquisition.

The procedure for synthesis and administration of ${ }^{18} \mathrm{~F}-\mathrm{FDG}$ has been described previously. ${ }^{8}$ Briefly, the patients received $10 \mathrm{mCi}$ of ${ }^{18} \mathrm{~F}$-FDG when awake and supine in a quiet room. Blood samples were collected from the radial artery for estimation of the arterial radioactive input function to the brain. Twenty to 30 interleaved scans were initiated 30 min postinjection, yielding a total of 30 images, each separated by 3.375 $\mathrm{mm}$. Procedures for definition of the rostral medial frontal region have also been described previously. ${ }^{8}$ The locations of the cingulate and rostral frontal gyrus areas were defined from anatomic scans, and this information was used to define this area in functional scans. Anatomic fiducial markings were placed as described below to ensure accurate anatomic registration in each case.

\section{Anatomic Imaging (CT/MR Scans)}

CT or MR scans were performed in 26 of 31 patients (17 had MR scans only, 4 had CT scans only, 5 had both MR and CT scans, and 5 had no usable imaging data). CT scans were acquired on a Picker 1200 SX machine, and MR studies on a Picker H.P. Scanner (0.5T). Best 
available CT or MR imaging studies were selected for comparison with the PET images. Ten of the MR and PET scans were studied incorporating anatomic fiducial markings to allow the precise superimposition of PET and neuroradiological data. CT/MR and PET studies were performed within an average of 7 days of each other. CT/MR scans were evaluated by a neuroradiologist (J.A.B.) blind to other clinical results. Anatomic regions congruent with the regions of interest studied in the PET scans were rated for degree of atrophy using CT or MR images. Nineteen anatomic areas were rated for volume alteration, including the lateral and third ventricles, cingulate gyrus, interhemispheric fissure, frontal lobe structures, temporal lobe structures, parietal lobe cortex occipital cortex, hippocampus, cerebellar structures, subarachnoid space, medulla, and pons. The atrophy in each area was rated as normal, mild, moderate, or severe.

\section{RESULTS}

The neuropsychological test results with focus on the tests of abstraction and problem solving are presented along with summary IQ data from the Wechsler Adult Intelligence Scale in Table 2.

The mean IQ level of these patients is not significantly different from the normative average for the population. ${ }^{16}$ The averages indicated in Table 2 for both neuropsychological tests (WCST and HCT) are within the impaired range as reflected in neuropsychological test norms and cut-offs. ${ }^{13,17}$ The WCST concepts attained by these patients are significantly fewer than the normative average (mean 5.2 concepts, SD $1.5 ; t=2.48, p<0.02, d f=106$ ). The Category Test mean score would be equal to a $\mathrm{T}$ Score of 36 , reflecting at least a mild degree of neuropsychological impairment. ${ }^{17}$ The variance in the number of errors on the HCT is large for this mean level, reflecting considerable differences among subjects in their efficiency on this test.

The results of the PET studies of local cerebral metabolic rate for glucose (LCMRglc) in the cingulate gyrus and rostral superior frontal area for patients are presented in Table 3.

Student's $t$ test comparison between subjects in this

Table 2. Neuropsychological Test Results in the 31 Male Chronically AlcoholDependent Patients in This Study

\begin{tabular}{lcc}
\hline & Mean & SD \\
\hline Wechsler Adult Intelligence Scale- & 96.5 & 11.5 \\
Revised Full Scale IQ & & \\
WCST concepts attained & 4.3 & 1.8 \\
HCT Errors & 73.5 & 26.3 \\
\hline
\end{tabular}

Table 3. ${ }^{18} \mathrm{~F}$-FDG PET LCMRgic Measured with ${ }^{18} \mathrm{~F}-\mathrm{FDG}$ and PET in the Cingulate Gyrus and Rostral Frontal Area for 31 Male Chronically AlcoholDependent Patients, and 18 Age- and Sex-Matched Controls

\begin{tabular}{|c|c|c|c|c|}
\hline & \multicolumn{2}{|c|}{$\begin{array}{c}\text { Patients } \\
\text { 31) }\end{array}$} & \multicolumn{2}{|c|}{$\begin{array}{c}\text { Controls } \\
18)\end{array}(n=$} \\
\hline & Mean & SD & $\begin{array}{l}M- \\
\text { ean }\end{array}$ & SD \\
\hline $\begin{array}{l}\text { Cingulate gyrus LCMRglc } \\
\text { (absolute, } \mathrm{mg} / 100 \mathrm{~g} / \\
\text { min) }\end{array}$ & 5.99 & 1.08 & 6.95 & 1.02 \\
\hline $\begin{array}{l}\text { Cingulate gyrus LCMRglc } \\
\text { (normalized to whole } \\
\text { brain slice) }\end{array}$ & 0.98 & 0.06 & 1.06 & 1.07 \\
\hline
\end{tabular}

study and age-matched control subjects shows no difference on absolute values of LCMRglc, but significantly reduced normalized cingulate LCMRglc in this alcoholic patient sample $(t=206 ; p<0.0001 ; d f=47)$. This result was consistent for patients examined on either PET scanner as described previously (normalized values reflect a ratio of ROI LCMRglc as the numerator and whole brain slice LCMRglc as the denomenator).

We computed Pearson product-moment correlations between: (1) two demographic measures (age and years of education); (2) number of years of heavy drinking and maximum daily drink consumption; (3) two independent measures of metabolism (LCMRglc) in frontal brain structure (superior frontal gyrus and cingulate gyrus); (4) ratings of atrophy/abnormality in three frontal brain regions (anterior frontal cortex, interhemispheric fissure, and cingulate gyrus); and (5) neuropsychological test performance on two concept-formation tasks known to be sensitive to disease or trauma-related dysfunction in frontal brain areas. These data are presented in Table 4.

These results indicate a statistically significant association between level of performance on the WCST, CT/MR rating of cingulate region atrophy, and PET-measured cingulate LCMRglc. A significant association was not found with the HCT as the behavioral measure. Other significant relationships reflect the effect of age on brain volume and the interrelationship of structural measures of brain volume in the various frontal areas. Similar significant results obtained when nonparametric correlational techniques (e.g., Spearman rho, Kendall Tau-B) were applied to the data. As before, these correlation

Table 4. Pearson Product-Moment Between Demographic, Drinking History, PET, and Structural Imaging (MR/CT) Measures for Alcoholic Patients

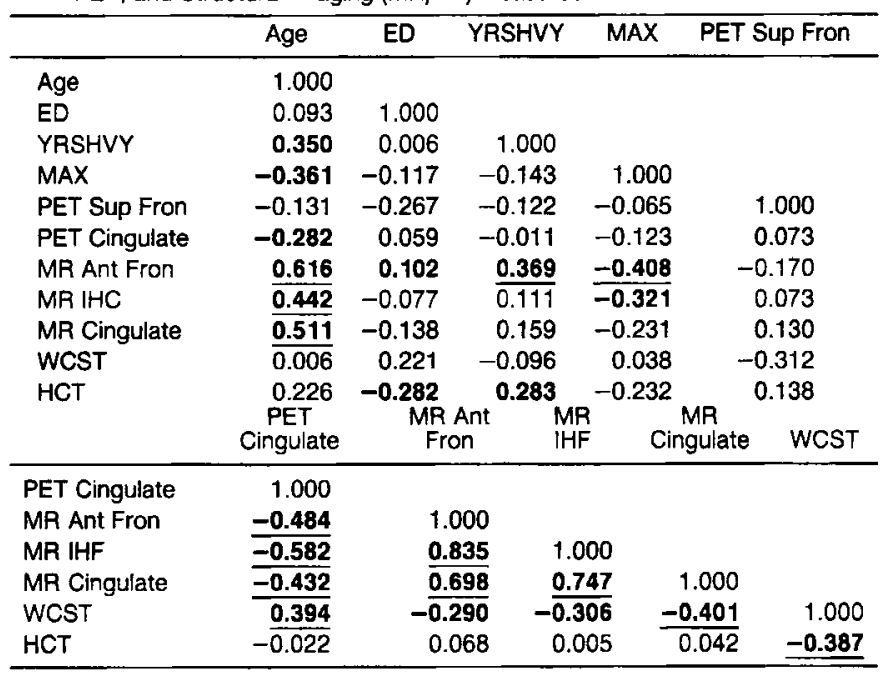

Age, chronological age of patient; ED, years of education for patient; YRSHVY, number of years of heavy drinking for patient; MAX, maximal daily drink consumption; PET Sup Fron, LCMRglc for superior frontal gyrus (normalized); PET Cingulate, LCMRglc for cingulate cortex (normalized); MR Ant Fron, MR/CT rating of anterior frontal cortex; MR IHC, MR/CT rating of interhemispheric fissure; MR Cingulate, MR/CT rating of cingulate cortex; WCST, Wisconsin Card Sorting Test categories achieved; CAT, HCT Errors. Bold figures indicate correlations $p<0.05$. Underlining indicates correlations $p<0.01$. 
results were found with both TCC and Siemens PET scanners.

Whereas the results of the correlational matrix can be understood by pairwise evaluation of the relationships of the variables, more graphic and multidimensional ways of understanding these results are of value. The relationship between age, normalized cingulate LCMRglc, and CT/ MR anatomic rating of atrophy is shown in Fig. 1. Reduced cingulate metabolism is associated with anatomic evidence of atrophy as a function of increasing age.

The relationship between decreased LCMRglc and increased cerebral atrophy in the cingulate region of the cerebral cortex is direct and significant $(r=-0.43 ; p<$ $0.01 ; d f=17)$. We examined the hypothesis that reduced cingulate LCMRglc would be associated with poorer performance on concept formation irrespective of the atrophic influence of atrophy as measured by structural imaging. A partial correlation matrix was used, including only patients studied on the Siemens PET Tomograph who had been examined with MR scans. The calculation was restricted to these cases because of the improved resolution of these instruments and the better potential for accurate registration between structural and functional images. This analysis revealed a significant association $(r$ $=0.54 ; p<0.01$ ) between concept formation performance on the WCST and cingulate LCMRglc when CT/MR rating of anatomic atrophy was held constant via statistical control. The same correlation was made for the HCT, but no significant result was found. Similar analyses conducted with other anatomic imaging variables did not produce significant neuropsychologic-metabolic relationships.

The relationship between cingulate CT/MR and WCST score in relation to age is shown in Fig. 2. The probability of poorer concept formation performance increases jointly as a function of increasing age and increasing degrees of cingulate atrophy.

The relationship between normalized LCMRglc, WCST score, and age is shown in Fig. 3. Although less clear in its interpretation, this graph suggests that reduced cingulate metabolic activity is generally associated with decrements

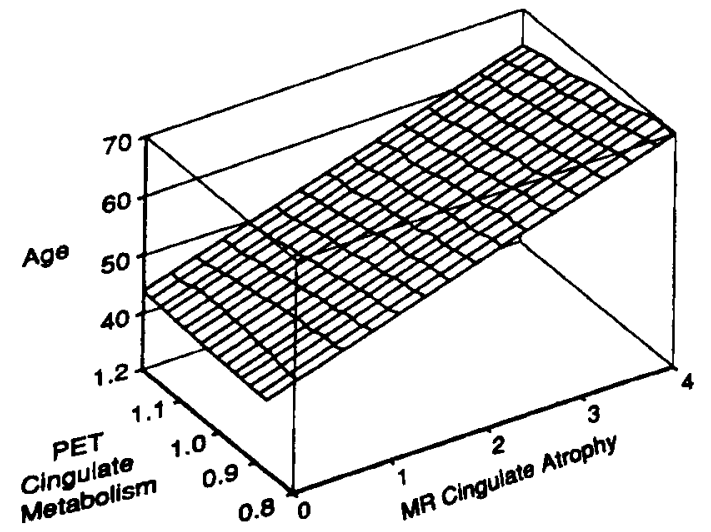

Fig. 1. Relationship between MR ratings of cingulate atrophy, normalized PET cingulate LCMRgIc, and age.

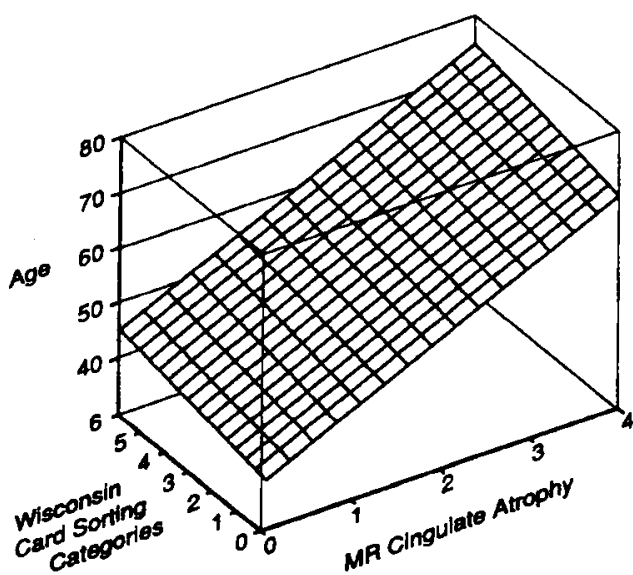

Fig. 2. Relationship between MR ratings of cingulate atrophy, Wisconsin Card Sorting Categories obtained by patients, and age.

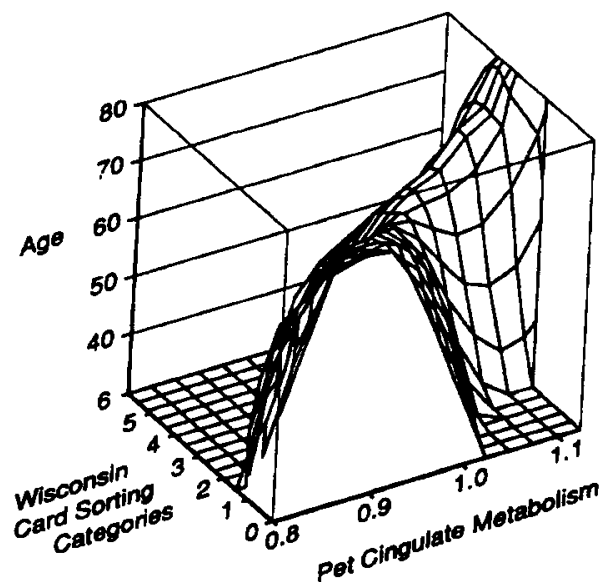

Fig. 3. Relationship between normalized PET cingulate LCMRgic, Wisconsin Card Sorting Categories obtained by patients, and age.

in concept formation achievement with increasing age in the chronic alcoholic patient.

\section{DISCUSSION}

This study demonstrates a significant correlation between degree of atrophy, metabolic functioning in the cingulate gyrus, and neuropsychological performance on one concept formation test in abstinent chronic alcoholic patients. Higher cerebral metabolic rates for glucose in the anterior cingulate gyrus are associated with better performance on the WCST, whereas lower rates are associated with poorer test performance. This relationship obtains in a more robust fashion with more advanced PET technology and MRI anatomic imaging; but the relationship between focal cingulate brain metabolism and a neuropsychological test sensitive to this general brain locus remains when atrophic effects from the same regional brain tissue areas are held constant.

Other studies ${ }^{18}$ have also reported a general decrease in glucose metabolic activity in alcoholics who are 50 years of age or older, and two previous studies demonstrated rostral frontal hypometabolism. ${ }^{7,8}$ This study extends these 
investigations by isolating a subarea of hypometabolism in the cortex that can be evaluated using a neuropsychological test of reasonable sensitivity and specificity for specific impairment. It is important to note that neuropsychological tests that are sensitive to frontal lobe disease or dysfunction are parametrically complex and can be affected by more generalized disorders when they are severe. $^{19}$

The results of this study indicate that a focal area of the brain seems to be vulnerable to the effects of chronic alcohol intake and its attendant risks (e.g., episodic malnutrition). This vulnerability does not seem to require the occurrence of specific alcoholic syndromes, such as delirium tremens, Wernicke-Korsakoff syndrome, or alcoholic cerebellar degeneration. In the present patients sample, 12 of the 31 patients showed evidence of alcoholic cerebellar degeneration, but none were diagnosed as having Wernicke-Korsakoff syndrome or delirium tremens on admission to hospital. The results may be of greater generalizability, because these patients are quite typical of many who come to treatment attention.

In the results presented herein, anatomic imaging and functional imaging studies both show the affected brain area, and in turn relate significantly to an objective behavioral test sensitive to dysfunction of that brain region. These findings offer confirmation of the widely accepted fact that patients with chronic alcoholism develop increasing problems with inductive logic, abstraction, and problem-solving with advancing age and unsuccessful treatment for their disease. ${ }^{20,21}$ Studies attempting to identify a cause for the deficit solely in anatomic imaging realms have met with only mixed success. ${ }^{5,22-24}$ The introduction of direct measures of metabolism offers greater potential for the integrated study of the chronic effects of alcohol abuse on the structure and functioning of the brain.

This study has several limitations. The number of cases is limited, although of moderate size for studies using PET technology. The results require cross-validation and confirmation. The methods for obtaining registration between PET and MR imaging regions of interest were careful, but cannot be seen as perfect. The area of hypometabolism studied here can be characterized as anterior cingulate in locus, but other parts of the medial frontal cortex may be affected as well. The pathogenesis of the disorder identified in this study is unknown, and there is no ready explanation why this particular area should be vulnerable, or how its relationship to other areas and systems in the brain relate in term of cause or effect. Finally, the use of two scanning machines did not prove to be a barrier when separate analysis of data from both scanners produced identical results, but data collected on the same apparatus would have been preferable.

The emergence of the WCST as a significant correlate in the analysis of relationships between imaging and behavioral variables is a noteworthy finding, but of equal interest is the failure of the Category Test to relate in the same way. There may be subtle differences in the responses of patients and characteristics of the two tests that may explain this discrepancy. The Category Test is generally held to be more difficult and rich in terms of its capture of "learning shifts," and this fact may have produced the rather large number of errors in these patients and the great variability in efficiency.

Clinicians will recognize that a subgroup of chronic alcoholic patients will manifest difficulties in treatment that are due to their lack of appreciation of the impact that advice and therapy should have on their lives. That is, patients will fail to see the importance of ideas and events in treatment much as they failed to recognize general "rules" and successful ways to cope effectively on the neuropsychological test in question. Unfortunately, the feedback in these situations may not be as immediate and direct as in the testing situation. Second, these results naturally raise the question of reversibility: Will metabolic and behavioral deficits respond to abstinence and more healthy living styles if treatment "takes hold"? The answer to the question is of importance for the patients' well being and for treatment planning in the longer term. Third, the results of this study may offer a challenge to clinicians who may want to work directly with patients to attempt to enable them to identify and circumvent problems that may occur as a result of their impairment. Little is known of the effectiveness of "cognitive remediation" strategies for alcoholic patients.

\section{REFERENCES} 1990

1. Lishman WA: Alcohol and the brain. Br J Psychiat 156:635-644,

2. Parsons OA, Butters N, Nathan PE (eds): Neuropsychology of Alcoholism: Implications for Diagnosis and Treatment. New York, Guilford, 1987

3. Grant I: Alcohol and the brain: Neuropsychological correlates. J Consult Clin Psychol 55:310-324, 1987

4. Tarter RE, Parsons OA: Conceptual shifting in chronic alcoholics. J Abnorm Psychol 77:71-75, 1971

5. Jernigan TL, Pfefferbaum A, Zatz LM: Computed tomography correlates in alcoholism, in Grant I (ed): Neuropsychiatric Correlates of Alcoholism. Washington DC, American Psychiatric Press, 1986, pp 2 I36

6. Jernigan TL, Butters N, DiTraglia G, Schafer K, Smith T, Irwin M, Grant I, Schuckit M, Cermak LS: Reduced cerebral gray matter observed in alcoholics using magnetic resonance imaging. Alcohol Clin Exp Res 15:418-427, 1991

7. Samson Y, Baron J-C, Feline A, et al.: Local cerebral glucose utilisation in chronic alcoholics: A positron emission tomography study. J Neurol Neurosurg Psychiat 49:1165-1170, 1986

8. Gilman S, Adams K, Koeppe RA, et al.: Cerebellar and frontal hypometabolism in alcoholic cerebellar degeneration studied with positron emission tomography. Ann Neurol 28:775-785, 1990

9. Adams KM, Grant I, Carlin A, Reed R: Self-reported alcohol consumption in four clinical groups. Am J Psychiatr 138:445-449, 1981

10. American Psychiatric Association. Diagnostic and Statistical Manual of Mental Disorders. Washington DC, American Psychiatric Association Press, 1987

11. Wesson D, Carlin A, Adams KM, Beschner G: Polydrug Abuse. New York, Academic Press, 1978

12. Adams $\mathrm{KM}$, Grant I: Influence of premorbid risk factors on 
neuropsychological performance in alcoholics. J Clin Exp Neuropsychol 8:362-370, 1986

13. Heaton RK: A Manual for the Wisconsin Card Sorting Test. Odessa FL, Psychological Assessment Resources, Inc., 1981

14. Reitan RM, Wolfson D: The Halstead-Reitan Neuropsychological Test Battery. Tucson, AZ, Neuropsychology Press, 1985

15. Fuster JM: The Prefrontal Cortex: Anatomy, Physiology, and Neuropsychology of the Frontal Lobe (2nd ed). New York, Raven Press, 1989

16. Wechsler D: The Wechsler Adult Inteligence Scale Manual. New York, The Psychological Corporation, 1981

17. Heaton RK, Grant I, Matthews CG: Comprehensive Norms for an Extended Halstead-Reitan Battery. Odessa FL, Psychological Assessment Resources, 1991

18. Sachs H, Russell JAG, Christman DR, Cook B: Alteration of regional cerebral glucose metabolic rate in non-Korsakoff chronic alcoholism. Arch Neurol 44:1242-1251, 1987
19. Robinson AL, Heaton RK, Lehman RAW, Stilson DW: The utility of the Wisconsin Card Sorting Test in detecting and localizing frontal lobe lesions. J Consult Clin Psychol 48:605-614, 1980

20. Tarter RE, Parsons OA: Conceptual shifting in alcoholics. J Abnorm Psychol 77:71-75, 1971

21. Grant I, Adams KM, Reed R: Aging, abstinence and medical risk factors in the prediction of neuropsychological deficit amongst chronic alcoholics. Arch Gen Psychiat 41:710-718, 1984

22. Ishii $\mathrm{T}$ : A comparison of cerebral atrophy in CT scan findings among alcoholic groups. Acta Psychiatr Scand 309(Suppl):7-30, 1983

23. Ron MA: The alcoholic brain: CT scan and psychological findings. Psychological Medicine, Monograph Supplement 3. Cambridge, Cambridge University Press, 1983

24. Wilkinson DA: Examination of alcoholics by computer tomographic (CT) scans: A critical review. Alcohol Clin Exp Res 6:31-45, 1982 